\title{
Première observation par l'expérience BABAR de la violation de symétrie de renversement du temps
}

Georges Vasseur (georges.vasseur@cea.fr)

Service de physique des particules, Irfu, CEA/Saclay, 91191 Gif-sur-Yvette Cedex

À notre échelle macroscopique, il est clair que les phénomènes ne sont pas invariants par renversement du temps.

Si, en tombant, un récipient se brise en mille pièces, on ne voit jamais les morceaux éparpillés au sol se rassembler ensuite spontanément pour former le récipient initial. On dit que la symétrie de renversement du temps n'est pas conservée.

\section{À l'échelle des particules élémentaires par contre,} les interactions fondamentales respectent généralement

la symétrie de renversement du temps. Seule l'interaction faible viole cette symétrie.

Restait à observer ce phénomène expérimentalement de manière directe. C'est ce que la Collaboration BABAR a réalisé en 2012.

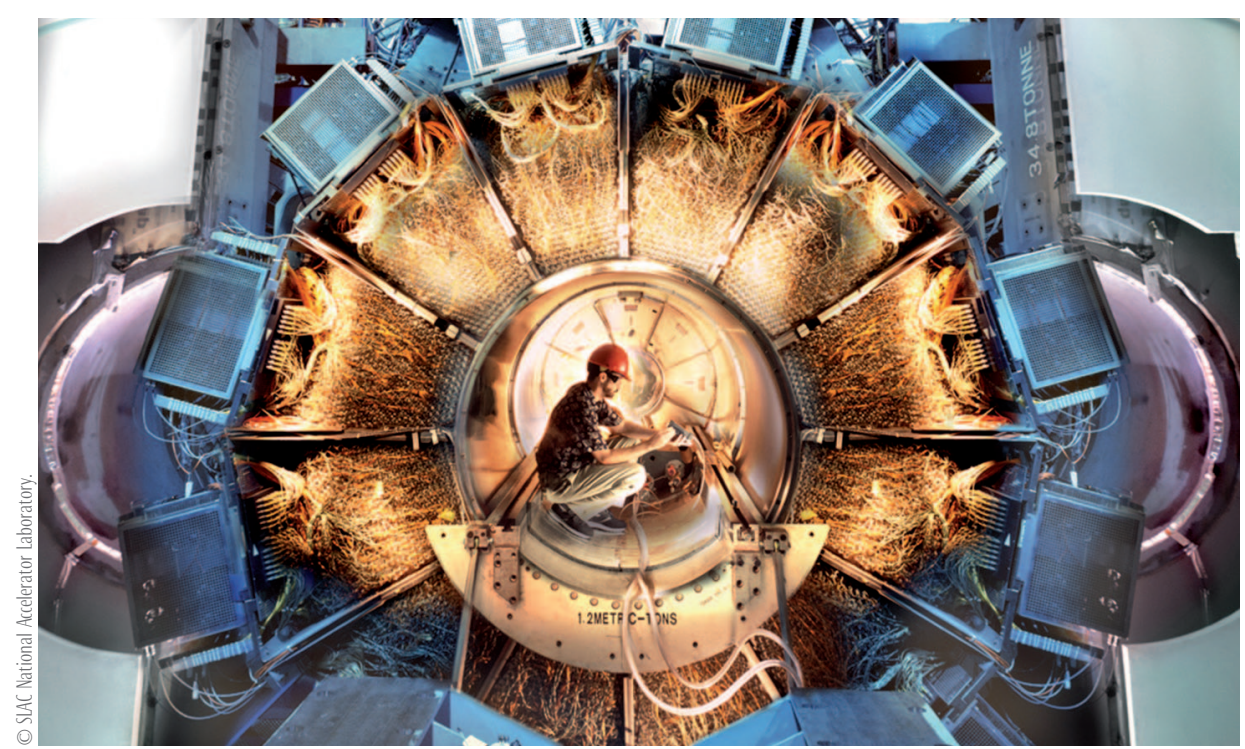

1. Vue transverse du détecteur BABAR. On aperçoit en particulier, en forme de pétales, les douze secteurs du détecteur DIRC d'identification des particules chargées, à la construction duquel les laboratoires français de l'expérience ont fortement contribué.

L'étude des symétries des systèmes et des interactions, en particulier de leur lien avec les lois de conservation, constitue une question fondamentale de la physique. Par exemple l'opération de renversement du temps, notée $\mathrm{T}$, consiste à dérouler un phénomène en inversant le sens d'écoulement du temps, ce qui revient à passer le film à l'envers. Elle est associée, en physique des particules élémentaires, à deux autres opérations de symétrie : la parité $\mathrm{P}$, qui inverse les coordonnées spatiales et équivaut, à une rotation près, à effectuer une symétrie dans un miroir, et la conjugaison de charge $\mathrm{C}$, qui transforme toute particule en son antiparticule. Pendant longtemps on a pensé que les équations fondamentales de la physique des particules étaient invariantes sous chacune de ces trois opérations. C'est le cas pour l'interaction électromagnétique et pour l'interaction forte (qui assure la cohésion des noyaux et des nucléons). Cependant, à la fin des années 1950, on découvre que l'interaction faible (responsable notamment de la radioactivité $\beta$ ) viole les symétries $\mathrm{P}$ et $\mathrm{C}$ de façon maximale ${ }^{(a)}$, tout en semblant conserver la symétrie produit CP. Une nouvelle surprise intervient en 1964, avec la découverte expérimentale d'une violation de la symétrie $\mathrm{CP}^{(\mathrm{b})}$ pour un type particulier de mésons (particules constituées d'un quark et d'un antiquark), les kaons électriquement neutres.

Il faudra attendre 2001 pour observer la violation de CP dans un autre système, celui des mésons $\mathrm{B}^{(c)}$ électriquement neutres, prouvant que ce phénomène n'est 
$>>$

pas limité au système des kaons mais est une caractéristique générale de l'interaction faible. C'est là l'œuvre des deux expériences concurrentes BABAR [1] (fig. 1) et BELLE, installées respectivement auprès des collisionneurs électron-positron PEP-II au laboratoire national de l'accélérateur SLAC (photo, p. 29) en Californie et KEKB au Japon, qui ont commencé à accumuler des données en 1999. Collisionneurs à énergies asymétriques, avec des faisceaux de grande intensité et une énergie dans le référentiel du centre de masse de la collision voisine de 10,58 GeV (correspondant à la masse de la résonance $\mathrm{Y}(4 \mathrm{~S})$, qui se désintègre en une paire constituée d'un méson $\mathrm{B}$ et de son antiparticule $\overline{\mathrm{B}}$ ), ce sont de véritables " usines à B ». En une décennie, ces deux expériences accumulent une statistique de plusieurs centaines de millions de paires $\mathrm{B} \overline{\mathrm{B}}$. En cherchant des différences de propriétés dans les désintégrations des mésons $\mathrm{B}$ d'une part et des mésons $\overline{\mathrm{B}}$ d'autre part, les deux collaborations internationales entreprennent alors une étude exhaustive de la violation de la symétrie CP [2] et vérifient que celle-ci est expliquée par le mécanisme ${ }^{(\mathrm{d})}$ proposé dès 1973 par les théoriciens Makoto Kobayashi et Toshihide Maskawa, qui obtiennent pour cela le prix Nobel de physique en 2008.

Sous des hypothèses très générales, on peut démontrer que la symétrie globale CPT doit être conservée en théorie quantique locale des champs. Ce résultat, connu sous le nom de théorème CPT, n'a jamais été mis en défaut expérimentalement. Or, si la symétrie CPT est conservée, la violation observée de CP implique la violation de la symétrie T. Mais si la violation de CP est relativement simple à étudier, la mise en évidence de la violation de $\mathrm{T}$ est beaucoup plus subtile : il faut pouvoir inverser l'état initial et l'état final, ce qui n'est pas réalisable expérimentalement si l'état final est constitué des produits de désintégration.

Or, dans le système des mésons B électriquement neutres, il est possible de choisir comme situation initiale ou finale l'état d'un méson $\mathrm{B}$ à un instant donné. En effet, les deux mésons B produits dans la désintégration de la résonance $\mathrm{Y}(4 \mathrm{~S})$ sont dans un état quantique intriqué, c'est-àdire que leur évolution est liée aussi longtemps qu'aucun des deux ne s'est désintégré. Ainsi, la mesure de l'état dans lequel

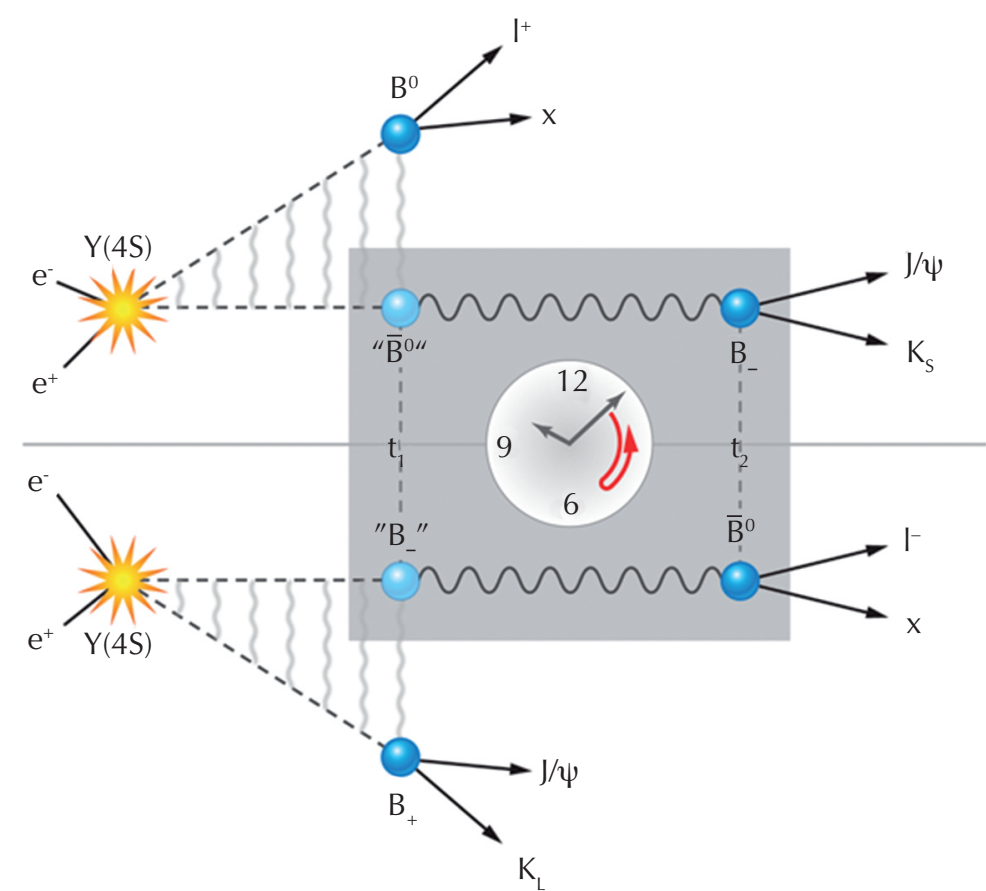

2. Deux processus symétriques l'un de l'autre par renversement du temps : (haut) $\overline{\mathrm{B}}^{0} \rightarrow \mathrm{B}_{-}$et (bas) $\mathrm{B}_{-} \rightarrow \overline{\mathrm{B}}^{0}$. En haut, le premier méson à se désintégrer est identifié comme l'état propre de saveur $\mathrm{B}^{0}$ par le signe positif du lepton issu de sa désintégration semi-leptonique, $\mathrm{B}^{0} \rightarrow \mathrm{I}^{+} \mathrm{x}$, à l'instant $\mathrm{t}_{\mathrm{f}}$. Le second méson est donc étiqueté en tant que méson $\bar{B}^{0}$ à cet instant $t_{1}$, tandis que sa désintégration en un état propre de $C P$ de valeur propre -1 , $\mathrm{J} / \psi \mathrm{K}_{\mathrm{S}}$, l'identifie comme méson $\mathrm{B}_{-}$à l'instant $\mathrm{t}_{2}$. En bas, on utilise le méson $\mathrm{B}_{+}$, caractérisé par sa désintégration en un état propre de $C P$ de valeur propre $+1, J / \psi K_{L}$, pour étiqueter le second méson comme $B$. à l'instant $t_{1}$, alors que son état $\bar{B}{ }^{0}$ à l'instant t t2 est identifié par sa désintégration semi-leptonique avec un lepton de charge négative, $\bar{B}^{0} \rightarrow I^{-} x$.

se trouve le premier méson B qui se désintègre à l'instant $t_{1}{ }^{(e)}$ nous renseigne sur l'état du second méson au même instant. Si, par exemple, le premier méson se désintègre comme l'état propre de saveur $\mathrm{B}^{0}$ (voir encadré), le second est nécessairement dans l'état propre de saveur opposée $\overline{\mathrm{B}}^{0}$ à cet instant. De même, si le premier méson se désintègre comme l'état propre de $\mathrm{CP}$ de valeur propre $+1, \mathrm{~B}_{+}$, le second est forcément dans l'état propre de CP de valeur propre opposée -1, B_, au même instant. Cependant, le second méson B, désormais seul, peut ensuite évoluer librement depuis cet instant $t_{1}$ jusqu'à l'instant $t_{2}$ où lui-même se désintègre. C'est justement cette évolution que la Collaboration BABAR a utilisé pour étudier la violation de la symétrie $T$.

Le principe de l'analyse est schématisé sur la figure 2. On considère l'évolution du méson B se désintégrant en dernier. Son état final est déterminé par son mode de désintégration, tandis que son état initial est déduit du mode de désintégration de l'autre méson B dans l'événement. On construit ainsi des processus transformés l'un de l'autre par renversement du temps, c'est-àdire en inversant l'état initial et l'état final, comme $\overline{\mathrm{B}}^{0} \rightarrow \mathrm{B}_{-}$et $\mathrm{B}_{-} \rightarrow \overline{\mathrm{B}}^{0}$ dans l'exemple de la figure 2. C'est en mesurant l'asymétrie, allant jusqu'à 70\%, des taux d'événements entre de tels processus symétriques par $\mathrm{T}$, que la Collaboration $\mathrm{BABAR}$ a mis en évidence, avec un niveau de confiance de 14 écarts standards, la violation de l'invariance par renversement du temps.

La publication de cette première observation directe de la violation de la symétrie par renversement du temps [3] a fait la couverture de Physical Review Letters le 19 novembre 2012. Le 14 décembre 2012, Physics World à son tour l'a incluse, classée en troisième position, dans sa liste des dix percées scientifiques mondiales en physique en 2012 [4]. En utilisant le phénomène d'intrication quantique, une observation directe de la violation de l'invariance par renversement du temps en utilisant des particules instables, longtemps considérée comme impossible, a été finalement réalisée par la Collaboration BABAR. 


\section{Références}

1- Site internet de l'expérience BaBAR: www-public.slac. stanford.edu/babar/. Cinq laboratoires français contribuent à l'expérience, quatre du CNRS/IN2P3 : le LAL à Orsay, le LAPP à Annecy, le LPNHE à Paris et le LLR à Palaiseau, ainsi que le CEA/Irfu à Saclay.

2 • "La violation de (P ", J. Chauveau et R. Granier de Cassagnac, Images de la physique, CNRS (2001-2002) 92-98

3• "Observation of Time-Reversal Violation in the $\mathrm{B}^{0}$ Meson System", J.P. Lees et al. (BABAR Collaboration), Phys. Rev. Lett. 109 (2012) 211801.

4• Liste complète sur le site internet du magazine Physics World : http://physicsworld.com/cws/article/ news/2012/dec/14/physics-world-reveals-its-top-10breakthroughs-for-2012

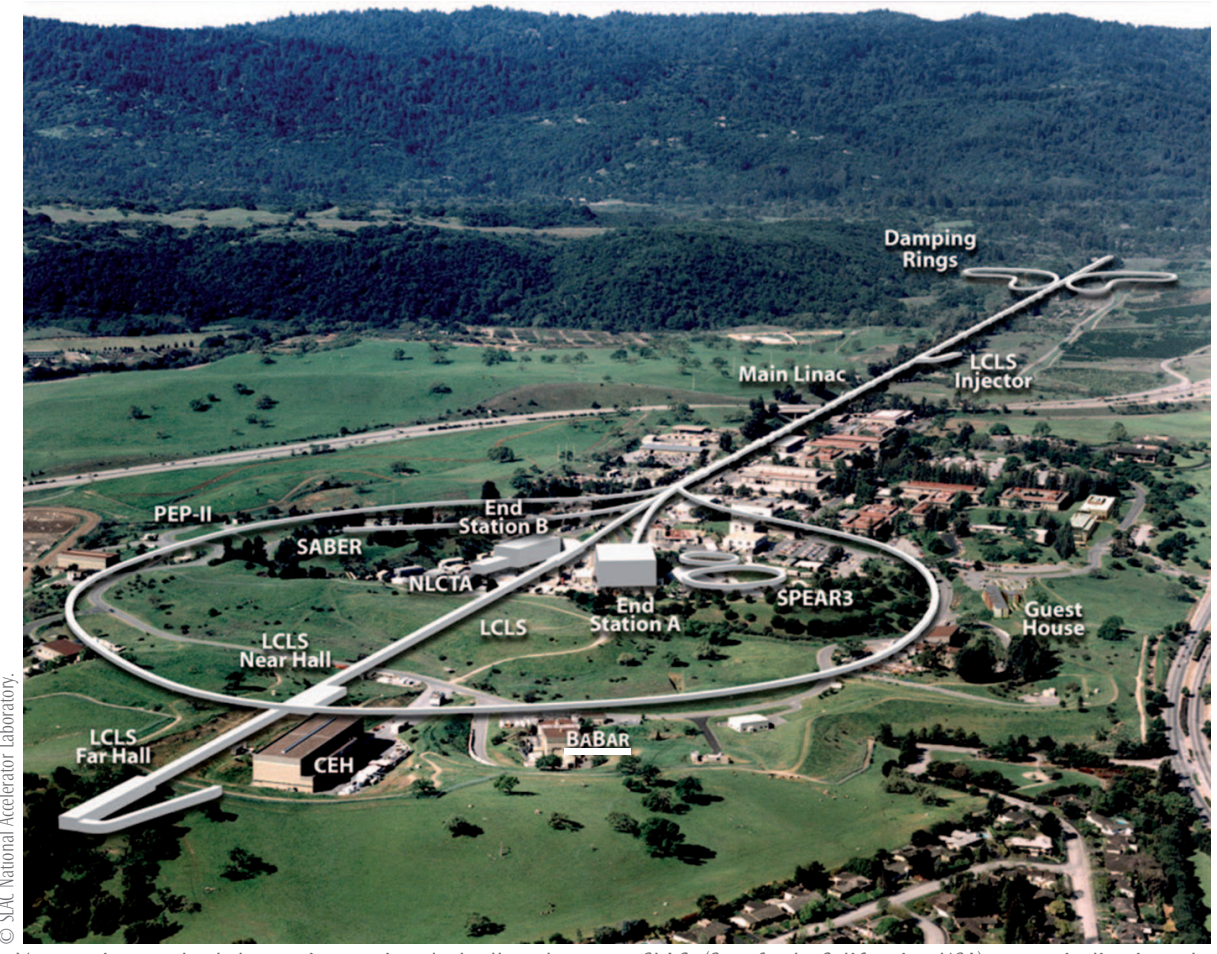

Vue aérienne du Laboratoire national de l'accélérateur SLAC (Stanford, Californie, USA), avec indication des expériences. L'expérience BABAR est située sous l'anneau de collisions.

\section{Production, propagation et désintégration des mésons B}

(a) La violation des symétries $\mathrm{P}$ et $\mathrm{C}$ a été mise en évidence expérimentalement pour la première fois en étudiant la désintégration $\beta$ du noyau de cobalt $\mathrm{Co}^{60}$.

(b) On observe alors que le kaon neutre de longue durée de vie, $\mathrm{K}_{\mathrm{L}}$, considéré comme un état impair sous la transformation $\mathrm{CP}$, se désintègre dans environ $0,2 \%$ des cas en une paire de deux mésons $\pi$ chargés, qui est un état pair sous $\mathrm{CP}$.

(c) Les mésons $\mathrm{B}$ contiennent ou bien un quark de saveur b ou bien un antiquark de saveur $\bar{b}$. Les quarks existent sous six variétés différentes, appelées saveurs et notées $\mathrm{d}, \mathrm{u}, \mathrm{s}, \mathrm{c}, \mathrm{b}$ et $\mathrm{t}$, que l'on regroupe en trois familles $(\mathrm{u}, \mathrm{d}),(\mathrm{c}, \mathrm{s})$ et $(\mathrm{t}, \mathrm{b})$. À chaque quark correspond une antiparticule, nommée antiquark, notée $\overline{\mathrm{d}}, \overline{\mathrm{u}}, \overline{\mathrm{s}}$, $\bar{c}, \bar{b}$ ou $\bar{t}$

(d) En postulant l'existence, confirmée expérimentalement par la suite, d'une troisième famille de quarks, Kobayashi et Maskawa étendent la matrice $2 \times 2$ de mélange des quarks de Cabibbo en une matrice $3 \times 3$, dénommée depuis matrice de Cabibbo-KobayashiMaskawa ou matrice CKM. Le terme complexe présent dans cette matrice fournit un mécanisme pour la violation de CP.

(e) Les mésons $\mathrm{B}$ ont un temps de vie moyen très court de 1,5 ps, mais cependant mesurable. Comme l'énergie du faisceau d'électrons est choisie différente de celle du faisceau de positrons, les mésons B sont produits en mouvement dans le référentiel du laboratoire, et parcourent une distance de l'ordre du quart de millimètre. L'instant de désintégration est calculé à partir de la position mesurée du point de désintégration.
Lorsqu'un électron et un positron entrent en collision à l'énergie des « usines à B », ils peuvent s'annihiler pour former un quark de saveur $b$ et un antiquark de saveur $\bar{b}$, qui se matérialisent sous forme d'un état lié de ces deux particules, la résonance $\mathrm{Y}(4 \mathrm{~S})$. Très instable, celle-ci se désintègre en moins de $10^{-22} \mathrm{~s}$ de façon pratiquement équiprobable, soit en une paire de mésons $B$ électriquement chargés, $B^{+}$et $B^{-}$, soit en une paire de mésons $B$ électriquement neutres, $B^{0}$ et $\bar{B}^{0}$. Seuls les mésons $B$ neutres nous intéressent ici.

$L^{\prime}$ interaction faible ne conserve pas la saveur et permet le mélange $B^{0}-\bar{B}^{0}$. Sous l'effet de ce phénomène, un méson $\mathrm{B}^{0}$ peut se transformer en $\overline{\mathrm{B}}^{0}$ et vice versa, de sorte que l'état d'un méson $B$ électriquement neutre, au cours de sa propagation, est une combinaison linéaire, variant avec le temps, des états $B^{0}$ et $\bar{B}^{0}$. Ces derniers sont les états propres de saveur. Leur contenu en termes de saveurs de quarks est parfaitement défini, $\bar{b} d$ pour le

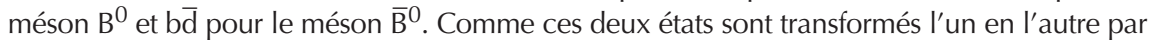
I'opération $C P$, les combinaisons linéaires particulières $B_{+}=\left(B^{0}+\bar{B}^{0}\right) / \sqrt{ } 2$ et $B_{-}=\left(B^{0}-\bar{B}^{0}\right) / \sqrt{ } 2$ sont les états propres de $\mathrm{CP}$ avec pour valeurs propres respectives +1 et -1 . (Ne pas confondre ces états propres de $\mathrm{CP}, \mathrm{B}_{+}$et $\mathrm{B}_{-}$, qui sont des mésons électriquement neutres, avec les mésons chargés $B^{+}$et $B^{-}$!)

Finalement, le méson $B$ se désintègre de multiples manières. Certains modes de désintégration sont particulièrement intéressants, car ils signent l'état quantique dans lequel se trouve le méson $B$ au moment de sa désintégration. $C^{\prime}$ est le cas des désintégrations semileptoniques, $\mathrm{B}^{0} \rightarrow \mathrm{I}^{+} \mathrm{x}$ et $\overline{\mathrm{B}}^{0} \rightarrow \mathrm{I}^{-} \mathrm{x}$, qui produisent, outre un ensemble de particules noté génériquement $x$, un lepton $I$, électron ou muon, dont le signe de la charge électrique est spécifique à la saveur du méson $\mathrm{B}$, positif pour $\mathrm{B}^{0}$ et négatif pour $\overline{\mathrm{B}}^{0}$. De même, un état final propre de CP identifie le méson B dans l'état propre de CP correspondant. Ainsi, les états $\mathrm{J} / \psi \mathrm{K}_{\mathrm{L}}$ et $\mathrm{J} / \psi \mathrm{K}_{\mathrm{S}}$ (le $\mathrm{J} / \psi$ est un méson formé d'un quark de saveur $\mathrm{c}$ et d'un antiquark de saveur $\overline{\mathrm{C}}$, tandis que les $\mathrm{K}_{\mathrm{S}}$ et $\mathrm{K}_{\mathrm{L}}$ sont deux formes de kaons neutres), de valeurs propres pour $\mathrm{CP}+1$ et -1 respectivement, signent la désintégration d'un méson dans l'état $\mathrm{B}_{+}$pour $\mathrm{J} / \psi \mathrm{K}_{\mathrm{L}}$, et $\mathrm{B}_{\text {_ pour }} \mathrm{J} / \psi \mathrm{K}_{\mathrm{S}}$. 